\title{
Application of Particle Swarm Optimization in Histogram Equalization for Image Enhancement
}

\author{
S. M. W. Masra, P. K. Pang, M. S. Muhammad, and K. Kipli \\ Department of Electronic Engineering, \\ Universiti Malaysia Sarawak (UNIMAS), \\ 94300 Kota Samarahan, Sarawak, Malaysia.
}

\begin{abstract}
The basic and most common technique for contrast adjustment in the image is using Histogram Equalization (HE). It is based on equalizing the histogram of the image and subsequently enhancing its contrast, and results in overall contrast improvement. This paper introduces a combination of normal HE technique and Particle Swarm Optimization (PSO) algorithm for enhancing distorted image naturally. The process is as follows. The image is separated into red, green and blue (RGB) channels and PSO algorithm is applied into each channel in order to get its best fitness value. The fitness value that is obtained then will be applied into HE's normalization, after that the processed color image will be merged back to RGB image. Experimental results have shown the effectiveness in improving the contrast of the original images without introducing disturbing artifacts caused by normal HE.
\end{abstract}

Keywords-image enhancement; histogram equalization; particle swarm optimization

\section{INTRODUCTION}

The purpose of image enhancement is to improve the interpretability or perception of information in images for human viewers. Basically, the idea behind this technique is to bring out the detail that is hidden in the image, or simply to highlight certain features of interest in the image. Histogram equalization (HE) is the basic and most common technique for enhancing the contrast in the image. It adjusts pixel intensities in order to obtain a new enhanced image. Through this adjustment, the intensities can be better distributed on a histogram. The histogram of the image with brightness levels is in the range of $[0,255]$. HE flattens and stretches the dynamic range of the resultant image histogram and as a consequences, it enhances the contrast of the image and gives an overall contrast improvement [1]. The advantage of this technique is it allows the poor contrast area gains a higher contrast. One drawback of the HE can be found on the fact that the brightness of the image can be changed after the HE, which is mainly due to the flattening property of the HE [2]. Many authors explored the use of Particle Swarm Optimization (PSO) to solve variety of problems in computer science and engineering [3, 4]. The application of PSO in image enhancement was presented in [5-7]. In [5], the author used PSO to solve the image enhancement problem by maximizing an objective fitness criterion in order to enhance contrast and detail in the image by adapting the parameters of a novel extension to a local enhancement technique. In [7], the authors proposed an enhancement technology of gray image where image enhancement is considered as an optimization problem and PSO is used to solve it. The author improved the quality of the intensity image by a parameterized transformation function, in which parameters are optimized by PSO based on an objective function.

In this paper, we have performed color image enhancement by a combination of normal HE and PSO algorithm. A program is developed using MATLAB software. The detail of the approach is discussed here. The paper is organized as follows: In Section II, PSO algorithm is described. In Section III, proposed methodology is discussed. In Section IV, results and discussions are presented, and finally in Section V, conclusions of the work are made.

\section{PARTICle SWARM OptIMIZATION}

Particle swarm optimization (PSO) is a new method of optimization developed in 1995 by Eberhart and Kennedy [7]. The idea of PSO was inspired by social behavior of bird flocking, fish schooling, as well as swarming theory. The concept of PSO is that each particle is flown in hyperspace or searched space to find its best solution (fitness) which is called pbest. Then, the overall best value (global value) called gbest achieved by any particle in the population with its position is identified [8].

In an optimization problem, the solution is associated to the position of the bird. This position is known as "particle". In order for the particle to move in the search space, it has its own space position and flying velocity. Each particle has the ability to memorize and track the current best particle (pbest) in a swarm. The particle then flies to the better position (gbest) based on its own best experience and its neighbors' best experience [9].

\section{PROPOSED Methodology}

In this research, HE with the application of PSO algorithm is used to process the test image in order to improve its quality. PSO task is to find optimal value which is known as the best fitness value.

A flowchart of the proposed method using HE with PSO application is shown in Fig. 1. Firstly, the three-channel red, green and blue (RGB) image is separated into Red, Green, and Blue channels. The initialization procedure includes insertion of PSO parameters and other values during calculation of 